\title{
AN IMPROVED ENERGY DEPOSITION MODEL IN MPACT AND EXPLICIT HEAT GENERATION COUPLING WITH CTF
}

\author{
Yuxuan Liu', Robert Salko², Kang Seog Kim ${ }^{2}$, Xinyan Wang', Matthew Kabelitz', \\ Brendan Kochunas ${ }^{1}$, Benjamin Collins ${ }^{2}$, William Martin ${ }^{1}$ \\ ${ }^{1}$ University of Michigan \\ 2355 Bonisteel Blvd., Ann Arbor, MI, USA, 48109 \\ ${ }^{2}$ Oak Ridge National Laboratory \\ One Bethel Valley Road, Oak Ridge, TN, USA, 37831
}

yuxuan1@umich.edu, salkork@ornl.gov,kimk1@ornl.gov,wxinyan@umich.edu, mkbz@umich.edu,bkochuna@umich.edu,collinsbs@ornl.gov,wrm@umich.edu

\begin{abstract}
The default energy deposition model in the CASL neutronics code MPACT assumes all fission energy is deposited locally in fuel rods. Furthermore, equilibrium delayed energy release is assumed for both steady-state and transient calculations. These approximations limit the accurate representation of the heat generation distribution in space and its variations over time, which are essential for power distribution and thermal-hydraulic coupling calculations. In this paper, an improved energy deposition model is presented in both the spatial and time domains. Spatially, the energy deposition through fission, neutron capture, and slowing-down reactions are explicitly modeled to account for the heat generation from all regions of a reactor core, and a gamma smearing scheme is developed that utilizes the gamma sources from neutron fission and capture. In the time domain, the delayed energy release is modeled by solving an additional equation of delayed heat emitters, similar to the equation of delayed neutron precursors. To allow the explicit heat generation coupling, the interfaces between MPACT and CTF were updated to transfer separate heat sources for different material regions (fuel, clad, moderator and guide tube). The results show that the distributions of the energy deposition between MPACT and MCNP agree very well for various 2-D assembly and quarter-core problems without TH feedback. The MPACT/CTF coupled calculation for the hot full power quartercore case exhibited a reduced peak pin power by $2.3 \%$ and a reduced peak fuel centerline temperature by $17 \mathrm{~K}$ when using the explicit energy deposition and heat transfer. The new model also shows a maximum $100 \mathrm{pcm} \mathrm{k}_{\text {eff }}$ effect on assembly depletion problems and an increased overall energy release by $7 \%$ in a PWR reactivity-initiated accident (RIA) problem.
\end{abstract}

KEYWORDS: Explicit energy deposition, delayed energy, gamma smearing, MPACT

\section{INTRODUCTION}

The energy released by a fission event consists of various energy modes. In addition to the direct fission energy release, the capture of neutrons via $(n, \gamma)$ or $(n, \alpha)$ reactions, for example, and the slowing down of neutrons also deposit energy. Most recoverable energy is released instantaneously in the forms of kinetic energy from fission fragments, fission neutrons, prompt gamma rays, or gamma rays from the capture of 
neutrons. About $7 \%$ of energy is released sometime after the fission event, from the radioactive decay of fission products, in the form of delayed beta and gamma rays. Equilibrium delayed energy release is reached after a period of steady power history, and usually the time dependence of the delayed energy is neglected in most reactor core simulations, except for estimating the decay heat during reactor shutdown. Table I provides the various energy release modes from neutron fission and capture, and the characteristics of these energy modes. To accurately model the energy deposition regarding the spatial and time dependence, special considerations are required for the boldface terms.

Table I. Fission energy release from ENDF/B-VII.1.

\begin{tabular}{|c|c|c|c|c|c|c|}
\hline \multicolumn{2}{|c|}{ Energy modes } & Fraction & $\mathrm{U}-235(\mathrm{MeV})$ & $\mathrm{U}-238(\mathrm{MeV})$ & Range & Time \\
\hline \multicolumn{2}{|c|}{ Fission fragments } & $80 \%$ & 169.13 & 169.80 & Local & Instant \\
\hline \multicolumn{2}{|c|}{ Beta decay of FPs } & $3-4 \%$ & 6.50 & 8.48 & Local & Delayed \\
\hline \multirow{2}{*}{$\begin{array}{l}\text { Fission } \\
\text { gammas }\end{array}$} & Prompt & $3-4 \%$ & 6.60 & 6.68 & \multirow{2}{*}{ Global } & Instant \\
\hline & Delayed & $3-4 \%$ & 6.33 & 8.25 & & Delayed \\
\hline \multicolumn{2}{|c|}{ Anti-neutrinos } & $4-5 \%$ & 8.75 & 11.39 & Unrecoverable & Delayed \\
\hline \multicolumn{2}{|c|}{ Neutron capture* } & $3-5 \%$ & \multicolumn{2}{|c|}{$6.0-11.0$} & Global & Instant/delayed \\
\hline \multicolumn{2}{|c|}{ Neutron scattering } & $2-3 \%$ & \multicolumn{2}{|c|}{$4.0-5.0$} & Global & Instant \\
\hline
\end{tabular}

*Energy released by capture of neutrons of all absorbers in the system via $(n, \gamma)$ and $(n, \alpha)$ reactions.

Currently, MPACT uses the effective isotopic fission energy release for core operation calculations [1]. The effective energy release includes an equilibrium delayed beta and gamma energy, and an averaged energy release contribution from neutron capture and slowing-down. Given a reactor thermal power $P$, the neutron flux is normalized by a factor $f$ determined in the following equation,

$$
P=f \sum_{j} V_{j} \sum_{i s o} \tilde{\kappa}_{f, i s o} N_{i s o, j} \sum_{g} \sigma_{f, g, i s o, j} \phi_{g, j}
$$

Where $\tilde{\kappa}_{f, \text { iso }}=$ effective fission energy release for isotope iso (unit: $\mathrm{J}$ ),

$V_{j}=$ volume of material region $j$ (unit: $\mathrm{cm}^{3}$ ),

$N_{i s o, j}=$ number density of isotope iso in material region $j$ (unit: $\mathrm{cm}^{-3}$ ),

$\sigma_{f, g, i s o, j}=$ microscopic fission cross section (unit: $\mathrm{cm}^{2}$ ), and

$\phi_{g, j}=$ neutron flux of energy group $g$ in region $j$ (unit: $\mathrm{cm}^{-2} \mathrm{~s}^{-1}$ ).

When the summation of material regions applies to a pin cell, Eq. (1) computes the pin power. Therefore, MPACT assumes that all the energy is deposited locally at the fission sites, i.e., inside the fuel rods. To consider the heat directly generated in the moderator via neutron and gamma reactions, a direct moderator heating fraction (user-defined) is presently used when coupled with CTF for TH calculation.

Several limitations of this energy deposition model include: (1) time dependence of the delayed energy modes is not considered in the time-dependent problems, such as depletion and transient problems; (2) a typical value of the neutron capture and slowing-down heat is built into fission kappa without considering the fuel composition explicitly; (3) all heat generation is assumed to be deposited in fuel rods when computing the pin power; and (4) no gamma smearing effect is considered. In this paper, an improved energy deposition model is developed in MPACT to eliminate these assumptions. 


\section{IMPROVED ENERGY DEPOSITION MODEL}

\subsection{Time-Dependent Energy Deposition Model}

Accounting for the time dependence of energy deposition involves two considerations. On one hand, in depletion, the overall effective energy release per fission varies with the isotopic concentrations via fission and capture reactions. As will be shown shortly, this effect is considered in the new model by accounting for the fission and capture energy releases explicitly. On the other hand, the time dependence of the delayed energy modes should be modeled for depletion and transient calculations. To proceed, the thermal power of a reactor, $P(t)$, is written as the sum of a prompt component, $P_{p r}(t)$, and a delayed component, $P_{d e}(t)$,

$$
P(t)=P_{p r}(t)+P_{d e}(t) .
$$

For a fissionable isotope, a fraction $\gamma_{i s o}^{f}$ of the fission energy is released sometime after the fission event through fission product decay. Similarly, the delayed fraction $\gamma_{i s o}^{c}$ of the energy for neutron capture can be defined, so $P_{p r}(t)$ is written as

$$
P_{p r}(t)=f(t) \sum_{j} V_{j} \sum_{i s o} N_{i s s, j}(t)\left[\begin{array}{l}
\left(1-\gamma_{i s o}^{f}\right) \kappa_{f, i s o} \sum_{g} \sigma_{f, g, i s o, j} \phi_{g, j}(t)+ \\
\left(1-\gamma_{i s o}^{c}\right) \kappa_{c, i s o} \sum_{g} \sigma_{c, g, i s s, j} \phi_{g, j}(t)+\sum_{g} \sum_{g^{\prime}} \sigma_{s, g^{\prime} \rightarrow g, i s o} \phi_{g^{\prime}, j} \Delta E\left(g^{\prime} \rightarrow g\right)
\end{array}\right] .
$$

In Eq. (3), the energy release due to neutron fission, capture, and slowing-down are explicitly modeled; this corresponds to the three terms in the bracket. $\kappa_{f, i s o}$ is the direct fission kappa without the contribution from fission neutrons (and the subsequent energy due to neutron capture and slowing down). $\kappa_{c, s o}$ is the averaged energy release due to neutron capture of isotope iso. $\Delta E\left(g^{\prime} \rightarrow g\right)$ is the average energy loss of neutrons scattered from group $g$ ' to $g$. Since the nonelastic scattering effect is small in LWR applications, all the slowing-down heat is assumed to be promptly released. Also, the dependence of $\kappa_{f, \text { iso }}$ on incident neutron energy is neglected. In fact, the energies of delayed gamma and beta are slightly dependent on incident neutron energy. According to the correlation in Chadwick [2], a fission from a $1 \mathrm{MeV}$ neutron or a thermal neutron could cause no more than $0.15 \mathrm{MeV}$ energy difference of delayed gamma and beta. This is within 1 $\sigma$ uncertainty of the ENDF fission energy release data. For a thermal fission system, the effect is even smaller.

To determine the delayed power $P_{d e}(t)$, a common approach is to lump the numerous components of the delayed heat source into a relatively small number of groups $[3,4]$. This approach is similar to the treatment of delayed neutron precursors, but here the group is characterized by the half-lives of delayed heat emitters. If $D_{m, i s o, j}^{x}$ is defined as the concentration of the delayed heat emitters in group $m$ from isotope iso at region $j$ for induced reaction $x$ (fission or capture), the balance equation that $D_{m, i s o, j}^{x}$ satisfies is given as

$$
\frac{d D_{m, i s o, j}^{x}(t)}{d t}=f(t) V_{j} \gamma_{m, i s o}^{x} \kappa_{x, i s o} N_{i s o, j}(t) \sum_{g} \sigma_{x, g, i s o, j} \phi_{g, j}(t)-\lambda_{m, i s o}^{x} D_{m, i s o, j}^{x}(t)
$$

where $\gamma_{m, i s o}^{x}$ and $\lambda_{m, i s o}^{x}$ are the yield and decay constants of delayed heat group $m$, so that $\sum_{m} \gamma_{m, i s o}^{x}$ is equal to $\gamma_{i s o}^{x}$ in Eq. (3). By using $D_{m, i s s, j}^{x}$, the delayed power is calculated as 


$$
P_{d e}(t)=\sum_{j} \sum_{i s o} \sum_{x=f, c} \sum_{m} \lambda_{m, i s o}^{x} D_{m, i s o, j}^{x}(t) .
$$

To account for the time dependence of delayed energy, MPACT can solve Eqs. (2-5) instead of Eq. (1). The details of solving these equations can be found in another work of the authors [5].

\subsection{Spatially-Dependent Energy Deposition Model}

Most fission energy is deposited locally in fuel rods where fission events occur. However, a non-trivial fraction of the energy can also be deposited in the non-fuel regions, such as clad, moderator, control rods, and structural materials. By applying Eqs. (3) and (5) to a spatial cell, i.e., the summation over the material regions of the cell, the local heat can be estimated explicitly.

The spatial model should also account for the energy deposition of gamma rays, essentially from neutron fission and capture for thermal systems. Without solving the gamma transport equation, an approximate gamma smearing model was developed in this work based on the following rationale: (1) the spatial gamma energy sources can be estimated according to the fission and capture rates; (2) the gamma energy is assumed to be redistributed within a predefined block of $n \times n$ pin cells due to the longer mean free path of gamma rays; (3) "pseudo pins" with zero gamma source are placed in the radial reflector to consider the leakage effect; and (4) according to Knoll [6], the gamma interaction cross sections are essentially proportional to the power of isotope atomic number. As a result, Eq. (6) is used to compute the final pin power $\tilde{P}_{i, j}$,

$$
\tilde{P}_{i, j}=\left(1.0-S_{i, j}\right) P_{i, j}+\frac{W_{i, j}}{\sum_{i=1}^{n} \sum_{j=1}^{n} W_{i, j}} \sum_{i=1}^{n} \sum_{j=1}^{n} P_{i, j} S_{i, j},
$$

where $P_{i, j}$ is the initial power of pin $(i, j)$ before gamma smearing, $S_{i, j}$ is the fraction of gamma energy at pin $(i, j)$, including prompt and delayed gamma from fission and capture, $n$ is the number of pins on a side of the smearing block, and $W_{i, j}$ is the weighting function of pin $(i, j)$ evaluated as $W_{i, j}=\sum_{r} \sum_{i s o} Z_{r, i s o}^{l} N_{r, i s o} V_{r}$, where $Z$ is the atomic number of isotope iso in subregion $r$ of pin $(i, j), N$ is the number density of the isotope, and $V$ is the volume of the subregion. The coefficients $l$ and $n$ were determined by trial and error according to the MCNP gamma transport solutions of a set of 2-D assembly problems [7]. It was found that $l=1.6$ and $n=7$ result in good agreements for a range of 2-D assembly problems. Also, the Z-dependences of the photoelectric, Compton, and pair production cross sections are in the form of $\mathrm{Z}^{4-5}, \mathrm{Z}$, and $\mathrm{Z}^{2}$, respectively [6], so $Z^{1.6}$ is a reasonably approximated average because Compton scattering is the dominating gamma reaction in the reactor. Furthermore, only the 2-D indices $(i, j)$ were involved in Eq. (6), so the axial gamma smearing effect was neglected. When estimating the gamma power distribution within a pin cell, the weighting function in the same form is calculated for each material region.

\subsection{Explicit Heat Transfer between MPACT and CTF}

The current coupling between MPACT and CTF only considered the heat generated from fuel rods. The direct heating in the moderator was approximated by a user-defined fraction (normally about $2.6 \%$ ). The new energy deposition model could provide the time-dependent heat source of any material region of a pin cell. Therefore, an explicit coupling of these heat sources between MPACT and CTF was implemented by considering the following heat sources to be transferred to CTF: (1) the heat deposited in the fuel rod; (2) the heat deposited in the fuel clad; (3) the heat deposited in the moderator outside a fuel rod or a guide tube, including the heat deposited in the spacer grid; (4) the heat deposited inside the guide tube (everything 
inside the guide tube was counted). When explicit energy deposition was used, the direct moderator heating fraction was set to zero. Also, CTF currently does not model flow inside of the guide tube, so none of the heating in the guide tube led to heating inside the guide tube. All of the energy left the outer surface of the guide tube and entered flow channels that were adjacent to the guide tube.

\section{NUMERICAL RESULTS}

The new energy deposition model in MPACT was verified against an MCNP energy deposition benchmark [7] previously developed for the VERA Progression Problems [8], as described in Section 3.1. These problems were run with coupled neutron and photon transport with MCNP. The effects of the new energy deposition model on the coupled TH, depletion, and transient calculations are presented in Section 3.2.

\subsection{Verification of the Explicit Energy Deposition Model in MPACT}

A quick verification was performed first to ensure the overall effective kappa was preserved by using the explicit energy deposition. The total energy release and total fission rate were tallied in both MPACT and MCNP calculations for VERA Problems $2 \mathrm{~b}$ (regular $\mathrm{UO}_{2}$ assembly) and $2 \mathrm{p}\left(\mathrm{UO}_{2}\right.$ assembly with 24 gadolinia fuel rods). Since the energy deposition due to neutron capture was not explicitly modeled with the default MPACT model, there was a bias for both cases compared with MCNP. The results of explicit energy deposition are in good agreement with MCNP results, as shown in Table II.

Table II. Comparison of overall effective kappa* (MeV/fission).

\begin{tabular}{|c|c|c|c|}
\hline VERA Problem & MCNP & MPACT default & MPACT explicit model \\
\hline $2 \mathrm{~b}\left(\mathrm{UO}_{2}\right)$ & $199.37( \pm 0.02)$ & 202.84 & 199.63 \\
\hline $2 \mathrm{p}(\mathrm{Gad})$ & $202.17( \pm 0.02)$ & 202.95 & 202.24 \\
\hline
\end{tabular}

* The neutron scattering and capture related energy are $\mathrm{k}_{\text {eff-corrected for non-critical systems [7] }}$

To investigate the spatial effects, the default and explicit energy deposition models of MPACT were compared with the MCNP benchmark [7] for VERA Problem 2. It was identified in the MCNP benchmark calculation that several cadmium and indium isotopes (Cd-108, 110, 112, 113, 114, 116 and In-113, 115) of the silver-indium-cadmium (AIC) control rod do not have gamma-ray production data in the ENDF/BVII.1 based ACE libraries [9]. Therefore, the gamma rays induced by neutron reactions with these isotopes are not being transported in MCNP and the energy is deposited locally. To be consistent with MCNP for verification purpose, the energy of gamma rays of these isotopes are locally deposited in MPACT without performing the gamma smearing. Figure 1 shows the difference between the pin power results of two representative cases, one with AIC control rods (blue) and the other with gadolinia fuels (red). The pinwise energy deposition was normalized such that the average of all fuel pins is unity, and then that normalization factor was multiplied to the non-fuel pins. The powers of non-fuel pins were all zero for the default MPACT model, which led to notable differences when compared with the MCNP results. The explicit model accounted for the heat of the non-fuel regions with the maximum error less than $1.4 \%$. The fuel pin powers also improved due to the gamma smearing model $(<0.7 \%$ for both cases).

\begin{tabular}{|c|c|c|c|c|c|c|c|}
\hline-0.048 & & & \multirow{2}{*}{\multicolumn{5}{|c|}{ MPACT (default) - MCNP }} \\
\hline 0.012 & 0.005 & & & & & & \\
\hline-0.002 & 0.001 & -0.004 & & & & & \multirow{5}{*}{$2 g(A I C)$} \\
\hline-0.279 & -0.003 & -0.006 & -0.272 & & & & \\
\hline-0.005 & -0.004 & -0.008 & -0.006 & -0.010 & & & \\
\hline-0.004 & -0.006 & -0.005 & -0.007 & -0.012 & -0.285 & & \\
\hline-0.284 & -0.005 & -0.005 & -0.285 & -0.009 & -0.003 & 0.005 & \\
\hline-0.002 & 0.000 & -0.001 & -0.002 & -0.002 & 0.004 & 0.006 & 0.012 \\
\hline 0.008 & 0.008 & 0.010 & 0.011 & 0.006 & 0.009 & 0.008 & \begin{tabular}{|l|l|}
0.011 & 0.01 \\
\end{tabular} \\
\hline
\end{tabular}

\begin{tabular}{|c|c|c|c|c|c|c|c|c|}
\hline 0.001 & & & \multicolumn{6}{|c|}{ MPACT (explicit) - MCNP } \\
\hline 0.000 & -0.002 & -0.004 & & & & & & \\
\hline 0.011 & 0.003 & 0.002 & 0.014 & & & & & \\
\hline 0.004 & -0.001 & -0.004 & 0.006 & 0.000 & & & & \\
\hline 0.006 & -0.002 & 0.000 & 0.007 & 0.003 & 0.011 & & & \\
\hline 0.012 & 0.003 & 0.004 & 0.013 & 0.002 & 0.004 & 0.003 & & \\
\hline 0.000 & -0.003 & -0.003 & 0.001 & -0.005 & -0.002 & -0.004 & -0.002 & \\
\hline 0.002 & -0.001 & 0.003 & 0.004 & -0.003 & -0.001 & -0.004 & -0.004 & -0.004 \\
\hline
\end{tabular}




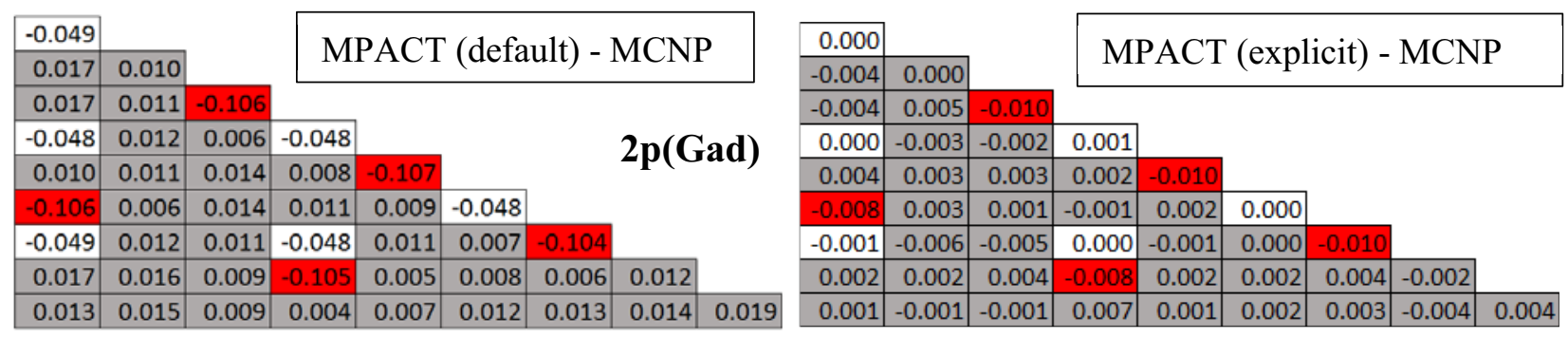

Figure 1. Comparison of energy deposition between MPACT and MCNP. Maximum standard deviation of MCNP results: 0.0017 .

A 2-D quarter-core problem, VERA Problem 5b-2D (with control rod Bank D inserted) is run to further confirm the energy deposition calculation in MPACT. Figure 2 shows that the explicit model was effective in producing the consistent energy deposition distribution as compared to MCNP, reducing the maximum error from $2.95 \%$ to $1.81 \%$. For comparison, the fission rate distributions of MCNP and MPACT had the maximum error of $1.85 \%$ (not shown in Figure 2), suggesting the remaining error of the energy deposition was mainly due to other sources of the transport calculation and the MCNP statistics.
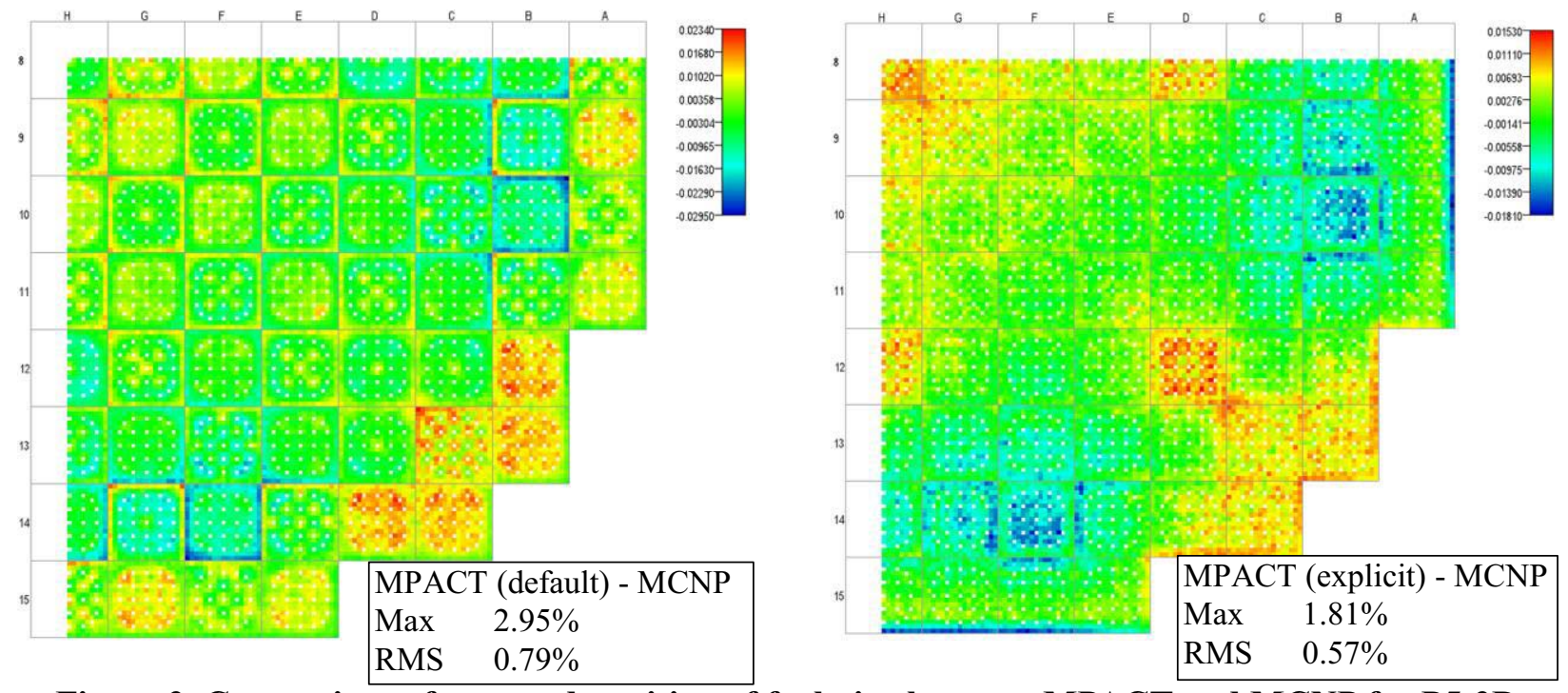

Figure 2. Comparison of energy deposition of fuel pins between MPACT and MCNP for P5-2D. Maximum standard deviation of MCNP results: $0.39 \%$.

\subsection{Effects of the Explicit Energy Deposition Model}

The comparisons of energy deposition models on spatial heat generations in the coupled steady-state, depletion, and transient calculations for a few typical PWR problems are discussed in the rest of the paper.

\subsubsection{Spatial heat generation and temperature distributions}

The coupling calculations of MPACT and CTF were run with VERA Problem 5b-2D using the default and the explicit energy deposition and heat transfers as discussed in Section 2. Figure 3 shows the fuel centerline temperatures of the 2-D core. The temperature difference of the explicit model was up to $20 \mathrm{~K}$ as compared to the default model, with the hottest pin temperature reduced by $17 \mathrm{~K}$. This is mainly due to the gamma smearing effect that reduced the peak pin power by $2.3 \%$. 

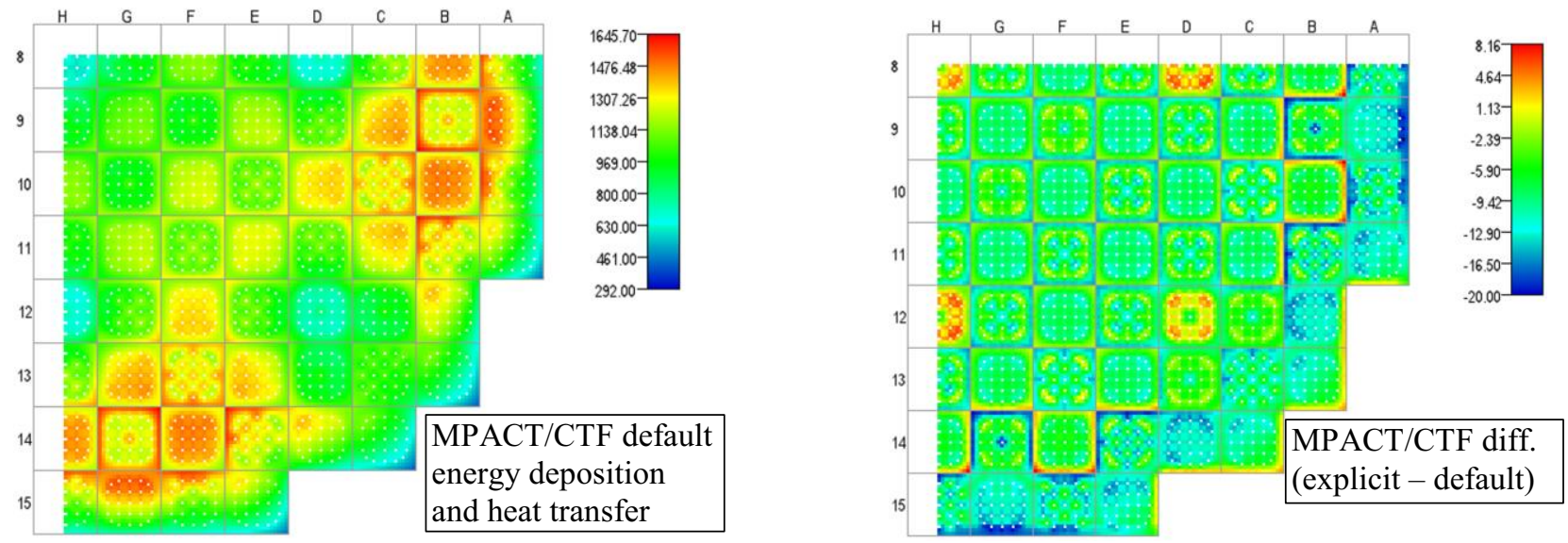

Figure 3. Fuel centerline temperatures of default MPACT/CTF heat transfer (left) and the difference of fuel centerline temperatures of explicit heat transfer (right).

\subsubsection{Effect of effective kappa in depletion calculation}

The estimation of the effective kappa affects the rate of depletion. As discussed in Section 3.1, the effective kappa should include the energy release of all reactions and should consider the time dependence of the delayed energy release. In a prior study, the time-dependent-delayed energy model did not show significant differences regarding the isotopics and $\mathrm{k}_{\text {eff }}$ as compared to the equilibrium delayed energy model [5]. This is because the time scale for the delayed energy modes to approach equilibrium was relatively small, leading to a negligible time-integrated effect on the depletion rate of isotopes.

However, modeling the energy release of all reactions had a non-trivial effect because of the variations of energy release from core-specific neutron capture by fission products. Figure 4 compares the spatially integrated effective kappa and $\mathrm{k}_{\text {eff }}$ between default and explicit energy deposition models in two burnup calculations: VERA Problems $2 \mathrm{~b}\left(\mathrm{UO}_{2}\right)$ and $2 \mathrm{p}(\mathrm{Gad})$. The default fission kappa in MPACT only included a typical contribution from neutron capture in an LWR reactor. The effective kappa from the explicit model increases faster with burnup by representing the true energy release from neutron captures by the fission products. The small dip of the effective kappa around $10 \mathrm{GWd} / \mathrm{tU}$ in $2 p$ is caused by the burn-out of the gadolinia isotopes. The maximum differences in $\mathrm{k}_{\text {eff }} \mathrm{were}$ around $100 \mathrm{pcm}$ for both cases, suggesting a nonnegligible effect of considering the energy deposition from neutron capture explicitly.
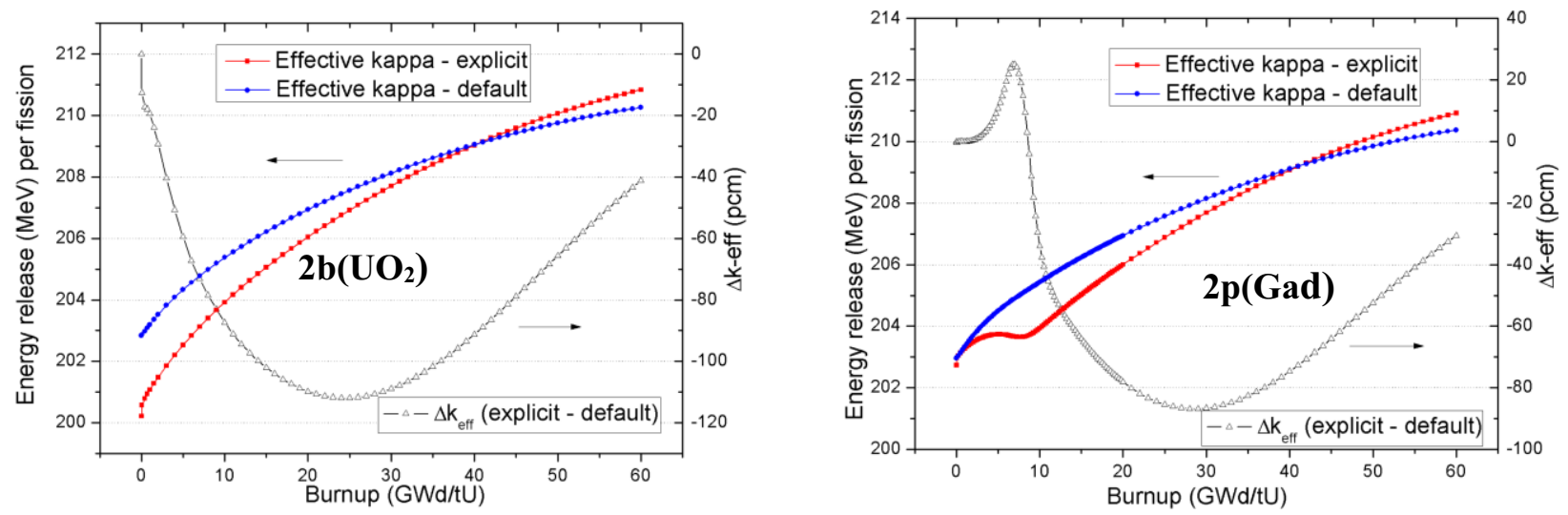

Figure 4. Effective kappa and $k_{e f f}$ in depletion for VERA Problem $2 b$ (left) and $2 p$ (right).

\subsubsection{Effect of delayed energy in fast transient calculation}


In fast transient calculations such as a reactivity-initiated accident (RIA), the time scale of the power excursion is often within a few seconds, so the delayed energy release significantly lags due to the drastic power change in a short time, suggesting a possibly larger effect when accounting for the time-dependentdelayed energy for such transients. As shown in the previous study [5], modeling the time-dependentdelayed energy for RIA problems proved to be important. For a PWR 3-D full core simulation of rod ejection, the peak core power was reduced by about $3 \%$, and the decay heat was significantly higher after SCRAM. The total energy release was predicted to be $7 \%$ greater by the time-dependent model because the delayed release of energy led to less Doppler effect, which increased the overall fission rates. Therefore, the equilibrium model is not conservative regarding the total energy release for fast transient simulations.

\section{CONCLUSIONS AND FUTURE WORK}

The explicit energy deposition model was developed in MPACT and the explicit heat transfer to CTF was implemented. Spatially, the energy deposition distributions calculated from the explicit model showed good agreement with those of MCNP neutron-photon coupled calculations for various VERA 2-D assembly and quarter-core problems. The MPACT and CTF coupled calculation for the quarter-core case exhibited a reduced peak pin power of $2.3 \%$ and a reduced peak fuel centerline temperature of $17 \mathrm{~K}$ when using the explicit energy deposition and heat transfer. The new model also showed a $100 \mathrm{pcm}$ maximum $\mathrm{k}_{\text {eff }}$ effect on depletion and an increase of overall energy release by $7 \%$ in an RIA simulation. Since the current gamma smearing approximation was tailored for LWR applications, ongoing work is being performed to develop the gamma transport capability that will run with the explicit energy deposition model.

\section{ACKNOWLEDGMENTS}

This research was supported by the Consortium for Advanced Simulation of Light Water Reactors (http://www.casl.gov), an Energy Innovation Hub (http://www.energy.gov/hubs) for Modeling and Simulation of Nuclear Reactors under US Department of Energy Contract No. DE-AC05-00OR22725. This research also made use of the resources of the High Performance Computing Center at Idaho National Laboratory, which is supported by the Office of Nuclear Energy of the US Department of Energy and the Nuclear Science User Facilities under Contract No. DE-AC07-05ID14517.

\section{REFERENCES}

1. K. Kim, et.al., "Neutron Capture Energies for Flux Normalization and Approximate Model for GammaSmeared Power," CASL-U-2017-1377-000, (2017).

2. M. Chadwick, "ENDF/B-VII.1 Nuclear Data for Science and Technology: Cross Sections, Covariances, Fission Product Yields and Decay Data," Nucl. Data Sheets, 112, pp. 2887-2996 (2011).

3. P. J. Turinsky, et. al., "NESTLE, Few-Group Neutron Diffusion Equation Solver Utilizing the Nodal Expansion Method for Eigenvalue, Adjoint, Fixed-source Steady-state and Transient Problems," Electric Power Research Center, North Carolina State University (1994).

4. M. A. Rathbun and D. P. Griesheimer, "Effect of Delayed Energy Release on Power Normalization in Reactor Depletion Calculations," Trans. Am. Nucl. Soc., 116, pp. $1144-1149$ (2017).

5. Y. Liu, B. Kochunas, W. Martin, and T. Downar, "Delayed Fission Energy Effect on LWR Normal Operation and Transients," Annals of Nuclear Energy, 128, pp. 84-93 (2019).

6. G. Knoll, Radiation Detection and Measurement, John Wiley \& Sons. Inc., New York City, USA (2000).

7. X. Wang, Y. Liu, W. Martin, K, Kim, "Energy Deposition Analysis for VERA Progression Problems by MCNP," Proceedings of PHYSOR 2018, Cancun, Mexico (2018).

8. A. Godfrey, "VERA Core Physics Benchmark Progression Problem Specifications," CASL-U-20120131-003, CASL (2014).

9. J. Collins, "Listing of Available ACE Data Tables (rev. 4)," LA-UR-13-21822, Los Alamos National Laboratory (2014). 